\title{
From Snoring to Happy Sleeping!!
}

\section{Sonam Sethi*, Anmol Kapoor and Amrit Kapoor}

Department of Orthodontics and Dentofacial Orthopedics, India

*Corresponding Author: Sonam Sethi, Department of Orthodontics and Dentofacial Orthopedics, India.
Received: July 13, 2021

Published: August 05, 2021

(C) All rights are reserved by Sonam Sethi., et al.

\begin{abstract}
Introduction: Obstructive Sleep apnea (OSA) is a sleep disorder in which breathing repeatedly stops and starts. Symptoms include snoring and feeling exhausted even after a full night's sleep. Treatment often includes lifestyle changes, such as weight loss and the use of a breathing assistance device at night, such as a continuous positive airway pressure (CPAP) machine. However, due to the bulky design of the CPAP machine and it being an unpleasant experience for the bed partners, the drive for a more compliant design has led to the eXciteOSA machine.

Objective: To elicit the advancement in the treatment of OSA.

eXcite OSA: The genioglossal muscle neurostimulation technology. The eXcite OSA is a daytime therapy that uniquely addresses the cause of snoring and mild sleep apnea by using safe electrical current to stimulate and improve muscle functions in the mouth and tongue. The genioglossal muscle neurostimulation technology. The eXcite OSA is a daytime therapy that uniquely addresses the cause of snoring and mild sleep apnea by using safe electrical current to stimulate and improve muscle functions in the mouth and tongue.

Conclusion: The eXciteOSA mouthpiece may not be the holy grail of the treatment of mild obstructive sleep apnea, but it has shown remarkable results so far that could inspire future treatments.
\end{abstract}

Keywords: eXciteOSA; Obstructive Sleep Apnea (OSA); Continuous Positive Airway Pressure (CPAP)

\section{Introduction}

Sleep apnoea is a sleep disorder characterized by abnormal pauses in breathing or instances of abnormally low breathing, during sleep. Diagnosed with an overnight sleep test called a polysomnogram, or "sleep study", it is of three forms: central (CSA), obstructive (OSA) and complex/mixed sleep apnea (a combination of central and obstructive). OSA being the most common.

OSA is characterised by perfect silence through two, three, or four respiratory periods, in which there are ineffectual chest movements and finally air enters with a loud snort, after which there are several compensatory deep inspirations. The condition can also lower the flow of oxygen to your organs and cause uneven heart rhythms.

Risk factors for sleep apnea [2]:

- Male

- $\quad$ Old age

- $\quad$ Black, hispanic, or native American

- Family history of sleep apnea

- Asthma

- Smoking 


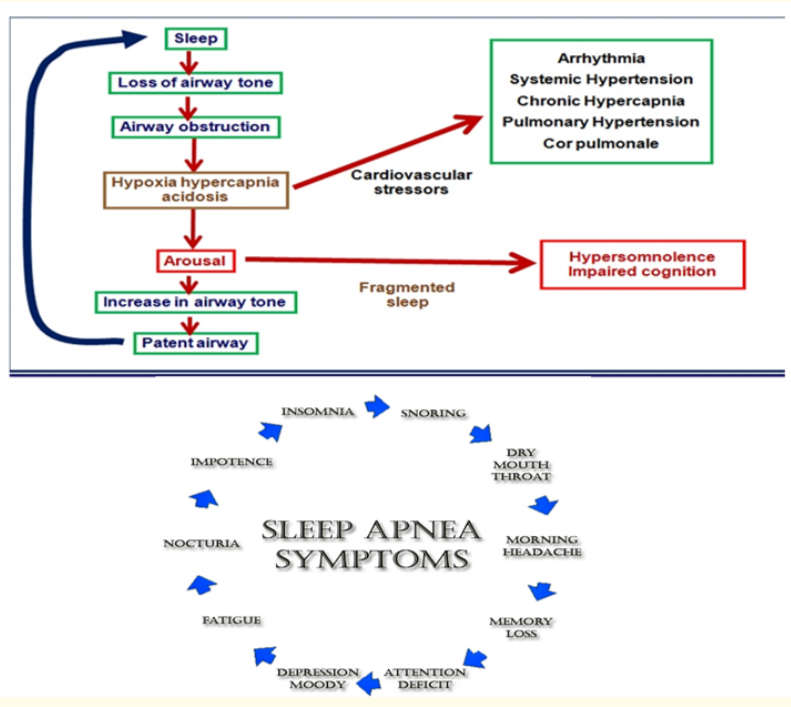

Figure 1: Pathophysiology and symptoms of OSA.

- Diabetes

- High blood pressure

- Cardiac diseases

- Obesity

- Large or thick neck

- Smaller airway: nose, throat, or mouth

- $\quad$ Large tongue.

\section{Complications of OSA [2]:}

- Sleepiness during the day and trouble concentrating: Adults may have a higher risk of accidents, and children may have a hard time at school.

- Cardiovascular problems such as heart attack, high blood pressure, unusual heart rhythms, or stroke.

- Eye problems such as glaucoma and dry eye.

- Metabolic disorders like type 2 diabetes.

- Problems with pregnancy like gestational diabetes or lowbirth-weight babies.

\section{Treatment of OSA:}

- Lifestyle changes: Weight loss, stop consumption of alcohol/ sleeping pills, sleep on the side, nasal sprays.
- Continuous Positive Airway Pressure (CPAP) machine: It is bulky therefore needs patient compliance.

- Oral devices for mandibular advancement to be worn while sleeping.

- Surgeries: Upper airway stimulator, Somnoplasty, $\mathrm{UP}_{3}$, Nasal surgery, Mandibular advancement.

Advancement in the treatment of OSA

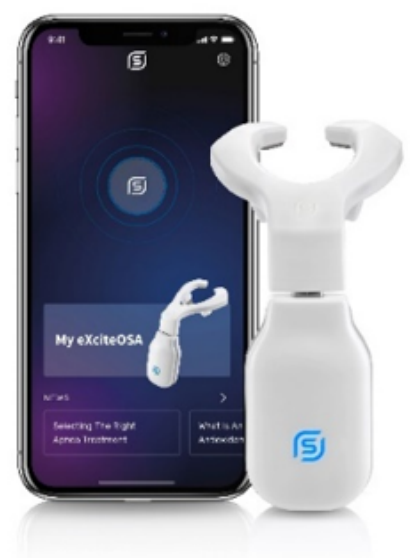

Figure 2: The eXciteOSA.

The genioglossal muscle neurostimulation technology wherein the eXciteOSA is a daytime therapy that uniquely addresses the cause of snoring and mild sleep apnea by using safe electrical current to stimulate and improve muscle functions in the mouth and tongue. The eXciteOSA mouthpiece uses four electrodes- 2 of which are located above the tongue and 2 of which are below the tongue. The strengthened tongue muscle no longer blocks the airway at night and thus enables tranquil sleep. The body can rest, refuel, and repair for the following day $[1,4]$.

\section{Easy treatment and monitoring [6-8]}

To be used for 20 mins for 6 weeks. Followed by twice a week for 20 mins to keep the tongue muscles in shape.

Monitoring is done on the eXciteOSA app.

\section{Advantages}

1. Day time treatment.

2. Not a bulky appliance. 


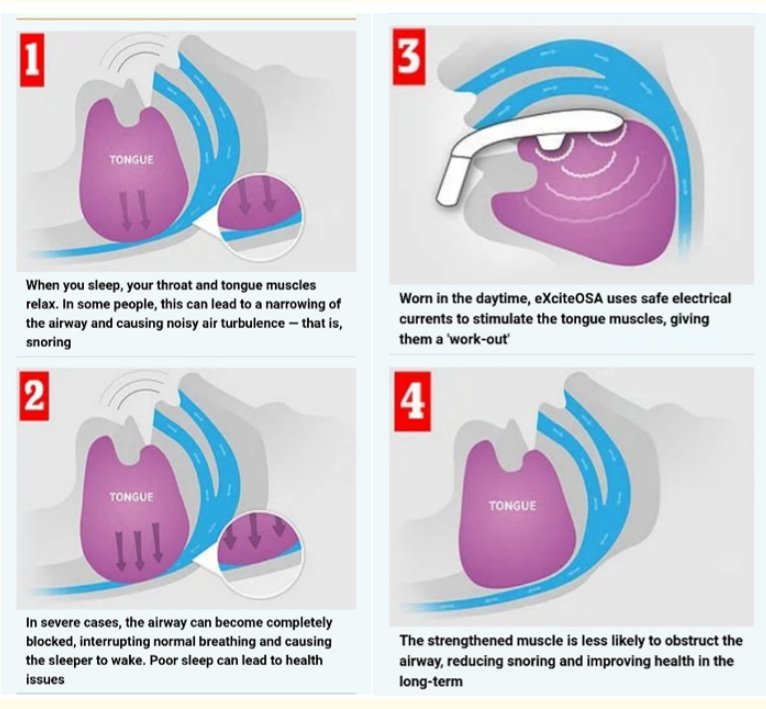

Figure 3: eXciteOSA in the mouth.

3. Not intrusive.

4. More compliant for the wearer and bed partners.

5. Costs similar to CPAP machine ( $£ 648)$.

\section{Side effects}

1. Excessive salivation

2. Tongue or tooth discomfort

3. Tongue tingling

4. Dental filling sensitivity

5. Metallic taste

6. Gagging

7. Tight jaw.

\section{Contraindications}

- $\quad$ Severe OSA (Apnea-hypopnea index level > 15)

- Patients with pacemakers or implanted pacing leads

- Pregnancy

- Patients with ulceration

- Ongoing orthodontic patients.

\section{Results [3,5]}

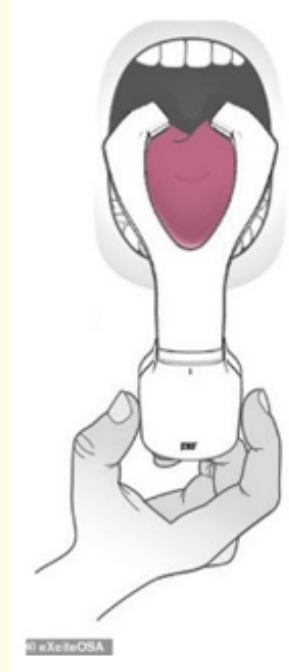

Figure 4

- In tests, FDA experts found that in the course of using the eX-citeOSA device, it reduced snoring of levels louder than 40 decibels, as loud as bird calls, by more than $20 \%$ in 87 of 115 patients.

- In a subset of patients who experienced snoring and mild obstructive sleep apnoea, the team noted an average reduction of $48 \%$ in the number of periods of shallow breathing per hour (from 10.2 to 5.3) in 41 out of 48 patients.

- In a study of 65 mild OSA patients, $79 \%$ of patients responded to eXciteOSA with a mean reduction of $52 \%$ in the Apnea Hypopnea Index, a 50\% reduction in the Oxygen Desaturation Index and a 3.9-point reduction in the Epworth Sleepiness Scale.

- In another significant study of eXciteOSA, the device exceeded the targeted endpoint of $20 \%$ mean reduction in snoring time, achieving nearly a $40 \%$ mean reduction in snoring time across 115 OSA patients.

\section{Conclusion}

The eXciteOSA mouthpiece may not be the holy grail treatment of mild obstructive sleep apnea, but it has shown remarkable results so far and could be a stepping stone for future treatments that try new methods of addressing these sleeping conditions. 


\section{Conflict of Interest}

Dr Sonam Sethi, Dr Anmol Kapoor, Dr Amrit Kapoor hereby declare that we have no conflict of interest.

\section{Bibliography}

1. Alyse Stanley. FDA Clears Tongue Stimulator You Use During the Day to Reduce Snoring and Sleep Apnea. Gizmodo (2021).

2. Conn Hastings. Intraoral Stimulation for Obstructive Sleep Apnea: Interview with Akhil Tripathi, CEO of Signifier Medical. Med gadget (2021).

3. Ian Randall. Ditch the earplugs! $£ 648$ device worn during the day sends electric currents to the TONGUE to strengthen muscles and cut snoring by more than $20 \%$ (2021).

4. Ditch the earplugs! $£ 648$ device worn during the day sends electric currents to the TONGUE to strengthen muscles and cut snoring by more than $20 \%$.

5. Matthew Gavidia. "FDA Clears First Daytime Treatment Device for Obstructive Sleep Apnea”. AJMC (2021).

6. Sama A., et al. "Daytime Intraoral Neurostimulation with Snoozeal ${ }^{\circledR}$ for Treatment of Snoring and Mild Sleep Apnea". CHEST Annual Meeting Notes (2018).

7. Signifier Medical ushers in new era of treatment for sleep apnea and snoring with FDA approval of eXciteOSA device". Boston, MA and London, UK: Signifier Medical Technologies (2021).

8. Wessolleck E., et al. "Intraoral electrical muscle stimulation in the treatment of snoring”. Somnologie 22.2 (2018): 47-52.

\section{Volume 5 Issue 9 September 2021}

(C) All rights are reserved by Sonam Sethi., et al. 